\title{
Particle Model for Quasibrittle Fracture aNd Application to Sea ICE
}

\author{
By Milan Jirásek ${ }^{1}$ and Zdeněk P. Bažant, ${ }^{2}$ Fellow, ASCE
}

\begin{abstract}
Fracture of quasibrittle materials with a large zone of distributed cracking is simulated by the particle model (discrete element method). The particles at the microlevel interact only by central forces with a prescribed force-displacement or stress-strain relation, which exhibits postpeak softening and is characterized by microstrength and microfracture energy. It is shown that a regular lattice, even though capable of closely approximating isotropic elastic properties, exhibits strong directional bias favoring propagation along a few preferred directions. A randomly generated particle model has no such bias. With a proper choice of the microlevel constitutive law, it can realistically simulate fracture of an ice floe during impact on a rigid obstacle. Explicit integration of the equations of motion is used to simulate the impact process and to explore the effect of the floe size and its initial velocity on the failure pattern and the history of the contact force.
\end{abstract}

\section{INTRODUCTION}

Fracture of quasibrittle structures can be analyzed relatively easily by simulating the heterogeneous microstructure with a particle system. The mechanical analysis of large systems of particles was initiated by Cundall (1971), Serrano and Rodriguez-Ortiz (1973), and Kawai (1980), who dealt with rigid particles that interact by friction and simulate the behavior of granular solids such as sand. This approach was developed and extensively applied by Cundall (1971) and Cundall and Strack (1979), who called it the distinct element method. An extension of Cundall's method to the study of microstructure and crack growth in geomaterials with finite interfacial tensile strength was introduced by Zubelewicz (1980, 1983), Zubelewicz and Mróz (1983), and Plesha and Aifantis (1983). As demonstrated by Zubelewicz and Bažant (1987), a particle model simulating the microstructure of an aggregate composite such as concrete can describe progressive distributed microcracking with gradual softening and with a large cracking zone. This model was modified and refined by Bažant et al. (1990), who used it in a study of the size effect on the nominal strength and on the postpeak slope of load-deflection diagrams. They showed that the spread of cracking and its localization can be realistically simulated. Observing that the macrofracture energy can hardly be determined by summing up the energies dissipated by fractured interparticle links, Bažant et al. (1990) showed that it can be determined by the size effect method. The particles were assumed to be elastic and have only axial interactions as in a truss. The interparticle contact layers of the matrix were described by a softening stress-strain relation. They also presented a method to generate the particle system randomly but with a prescribed particle size distribution. A similar approach, but with a more efficient numerical algorithm, was used by Jirásek and Bažant (1995) to determine the effect of the statistical coefficients of variation of microstrength and microductility on the mean fracture characteristics on the macroscale.

The discrete element method was also used by Belytschko et al. (1984), Dowding et al. (1991) and Plesha et al. (1991) for the modeling of dilatant slip in rock joints. These studies, which provided excellent descriptions of the complex behav-

\footnotetext{
'Postdoctoral Res. Fellow, Northwestern Univ., Evanston, IL 60208.

'Walter P. Murphy Prof. of Civ. Engrg. and Mat. Sci., Northwestern Univ., Evanston, IL.

Note. Associate Editor: Jean-Lou A. Chameau. Discussion open until February 1,1996 . To extend the closing date one month, a written request must be filed with the ASCE Manager of Journals. The manuscript for this paper was submitted for review and possible publication on August 27, 1993. This paper is part of the Journal of Engineering Mechanics, Vol. 121, No. 9, September, 1995. CASCE, ISSN 0733-9399/95/0009$1016-1025 / \$ 2.00+\$ .25$ per page. Paper No. 6864
}

ior of rock masses, did not consider transmission of tensile stresses across the joints and were not directly relevant to the fracture problem. Techniques related to Cundall's work were also exploited in studies of explosive impact and fragmentation [e.g., Thornton et al. (1993)].

The particle models are closely related to lattice methods, developed by theoretical physicists for the simulation of fracture processes in disordered materials (Disorder 1990; Herrmann 1991; Statistical 1990). Van Mier and Schlangen recently elaborated a lattice model for the static fracture of concrete. They used regular (Schlangen and van Mier 1992; van Mier and Schlangen 1993) as well as random (Schlangen 1993; Schlangen and van Mier 1993) frameworks, and a linear stressstrain law with a sudden stress drop and random strength to simulate a number of experiments. In the case of regular lattices or frameworks, however, one must suspect that the geometric regularity not reflecting the actual microstructure might favor fractures along the lattice lines. It is one purpose of the present study to explore this question.

Owing to its simplicity, the particle model is useful even for materials that do not consist of particles. It can serve as a convenient mathematical tool to force the cracking at the fracture front to be distributed over a large fracture process zone rather than localized into a line. It can thus impress onto the material in a very simple manner the nonlocal strainsoftening damage characteristics, the macroscopic continuum modeling of which is much more complicated. This idea will be exploited here for sea ice, in which there are, for the most part, no well-defined particles. Sea ice, however, is brittle and highly heterogeneous, and a material with such characteristics is generally known to exhibit large zones of distributed cracking that lead to macroscopic strain-softening behavior. The maximum particle size provides, from the viewpoint of macroscopic nonlocal continuum models, a characteristic length that governs the minimum width of cracking zones or strain-softening bands (this width is typically two to three times the maximum particle size). In the absence of particles, the characteristic length is determined by the maximum spacing of major inhomogeneities, and the particle model can be used to replace the role of such inhomogeneities. In sea ice, (a)

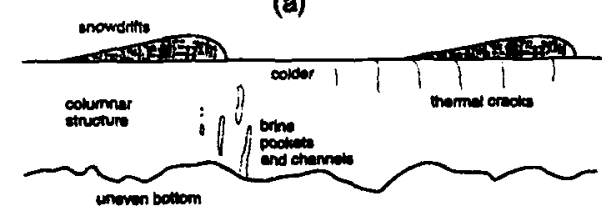

(b)

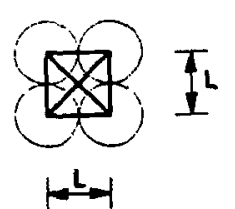

FIG. 1. (a) Major Inhomogeneities in Sea Ice Plates; (b) Basic Cell of Square Pattern 
small-scale inhomogeneities are the columnar grains and brinefilled voids and channels [Fig. 1(a)]. Large-scale inhomogeneities are thermal cracks (whose characteristic spacing is $0.3-1 \mathrm{~m}$ ), warm and cold regions caused by the alternation of snow drifts and bare ice (under a snow cover the ice surface is insulated and thus much warmer than bare ice), and unevenness of the bottom surface of the floating ice plate caused by water currents. Such inhomogeneities are the source of macroscopically nonlocal behavior.

The idea to analyze quasibrittle fracture by representing a structure as an assembly of particles with softening links can be extended to dynamic problems, e.g., to the impact of a moving ice floe on an obstacle. Due to its practical importance in the design of large offshore structures such as sea platforms or artificial islands, this problem has been receiving much attention in the literature on ice mechanics [see Proceedings 1992; Sanderson 1988]. However, most of the existing models have been based on rather simple assumptions such as an empirical relation between the contact area and the indentation pressure, while the fracture processes taking place outside the contact region have usually been neglected. A dynamic version of the particle model provides an attractive opportunity to explore possible applications of the discrete element approach in this area.

\section{BASIC EQUATIONS}

The structure is represented as an assembly of particles. The size of the particles should be proportional to the characteristic length of the material determined by the size of major inhomogeneities. For example, in the model for concrete developed by Bažant et al. (1990) and Zubelewicz and Bažant (1987), the particles represent large aggregates and are assumed to be perfectly rigid. Elastic deformation, damage, and fracture are reflected by changes in links connecting neighboring particles. The key ingredient of the model is the microlevel constitutive law-a law describing the behavior of an individual link. The simplest class of such laws assumes only axial interactions between particles, same as in a truss. Each link transmits an axial force $S$, the value of which can be determined from the history of the relative displacement $e$ between the two particles connected by the link. Depending on the spatial dimension, each particle has two or three degrees of freedom corresponding with the components of its displacement vector. This study focuses on the analysis of sea ice floes. All the modeling is two-dimensional (assuming plane stress in the floe).

The momentum equations for the particle system in matrix form read

$$
\mathbf{M d}+\mathbf{f}^{\mathrm{int}}=\mathbf{f}^{\mathrm{ext}}
$$

where $\mathbf{M}=$ mass matrix; $\mathbf{d}=$ vector of displacements; $\mathbf{f}^{\text {int }}$ $=$ vector of internal forces; $\mathbf{f}^{\mathrm{ext}}=$ vector of external forces; and a dot over a symbol denotes differentiation with respect to time. The components of $\mathbf{f}^{\mathrm{ext}}$ at the unsupported nodes are prescribed and they represent the applied loads. As the drag forces resulting from the difference between the motion of the ice floes and the motion of surrounding water and air are usually negligible compared with the contact forces, the external loads applied on the nodes are set to zero. However, the components of $\mathbf{f}^{\mathrm{ext}}$ corresponding with the displacements restricted by the condition of no penetration (discussed in later sections), represent the unknown contact forces between the floe and the obstacle.

Among many possible applications, this paper will deal with the impact of large sea ice floes on a rigid obstacle. The dimensions of ice floes observed in the Arctic Ocean are often in the order of several kilometers while the spacing of major inhomogeneities is only in the order of meters or tens of meters, and so a realistic particle model of a floe necessarily consists of a huge number of particles. Moreover, the particle interaction law must be strongly nonlinear. An efficient solution procedure in such a situation is the direct integration of (1) using an explicit method, e.g., the standard central difference method for second-order ordinary differential equations [e.g., Bathe (1982)]. In this method, based on the approximation $\ddot{\mathbf{d}}_{i}=\left(\mathbf{d}_{i+1}-2 \mathbf{d}_{i}\right) /(\Delta t)^{2}$ where the subscripts refer to discrete times $t_{i}(i=1,2, \ldots)$ with constant $\Delta t=$ $t_{i}-t_{i-1}$, one uses the simple recursive equations $\mathbf{d}_{i+1}=$ $2 \mathbf{d}_{i}-\mathbf{d}_{i-1}+(\Delta t)^{2} \mathbf{M}^{-1}\left(\mathbf{f}_{i}^{\mathrm{ext}}-\mathbf{f}_{i}^{\mathrm{int}}\right)$.

An important advantage of the explicit approach is that restrictions on the nodal displacements, such as those stemming from the condition of no penetration during an impact, can be easily incorporated into the algorithm. To this end, it is first assumed that all the nodes can move freely, the external forces are set to zero, the updated displacements are evaluated from the discretized equations of motion, and the condition of no penetration is checked for a specified set of nodes on the boundary that are candidates for collision with the obstacle. For example, if a floe is moving toward a cylindrical obstacle with radius $r_{0}$ centered at $\left(x_{0}, y_{0}\right)$, the condition of no penetration can be written as

$$
\left(x+d_{x}-x_{0}\right)^{2}+\left(y+d_{y}-y_{0}\right)^{2} \geq\left(r_{10}+r_{p}\right)^{2}
$$

where $x, y=$ initial coordinates of the particle center; $d_{x}, d_{y}$ $=$ its displacement components; and $r_{p}=$ radius of the particle (idealized as a circle). If this condition is satisfied no contact occurs, the computed displacement is correct, and the external force on this particle remains equal to zero. However, if contact is detected, the displacement components must be modified so as to satisfy condition (2) as an equality. The displacement is then considered as prescribed and the corresponding two equations of motion from (1) can be used to calculate the external force, which now represents the contact force between the obstacle and the particle. By summing up the external forces on all the particles currently in contact with the obstacle, the total impact force can be computed.

The only price to be paid for all the advantages of the explicit approach is the necessity to keep the time step sufficiently small. If a certain critical time step $\Delta t_{c}$ is exceeded, numerical instability occurs and completely invalidates the results. As is well known (Bathe 1982), the critical time step for a linear system without damping, $\mathbf{M d ̈}+\mathbf{K d}=\mathbf{f}^{\mathrm{cxt}}$, integrated by the central difference method, is $\Delta t_{c}=2 / \omega_{\max }$, where $\omega_{\max }$ is the highest circular eigenfrequency of the system. Of course, no general theorem of this kind is available for nonlinear systems, but experience shows that, unless the tangential stiffness of the system increases as it deforms, about $80 \%$ to $90 \%$ of the critical time step for a corresponding linearized system is usually satisfactory. The value of $\omega_{\max }$ can be estimated by representing the model as an assembly of truss elements with lumped mass points at their ends, and then using a theorem known from structural dynamics (Bathe 1982): all the eigenfrequencies of a finite-element system are less than or equal to the maximum eigenfrequency of its individual elements.

The issue of stability will be addressed in greater detail after the development of a specific geometrical arrangement and mechanical properties of the model.

\section{APPROXIMATING ISOTROPY WITH REGULAR LATTICE}

As no precise information on the spatial distribution of major inhomogeneities in ice plates is currently available, the particles are assumed to be of approximately the same size. 
First we explore a regular geometrical arrangement of the particles. The basic cell regularly repeated in the square pattern consists of four particles whose centers are located at the corners of a square and side length $L$ and are connected by four principal links and two diagonal links [Fig. 1(b)].

The mass of each particle and the microlevel constitutive law must now be specified. To preserve the total mass of a continuum with a mass density $\rho$, the mass of each particle must be $m=\mathrm{p} L^{2} h$, where $h$ is the thickness of the ice plate. In what follows, all the thickness-dependent quantities will be taken per unit thickness $(h=1)$, e.g., $m=\rho L^{2}$. The simplest form of the microlevel constitutive law for a fracturing material is a linear relationship between the force $S$ and the extension of the link $e$ with a sudden drop of the force at a certain tensile threshold $S_{p}$ [Fig. 2(a)]. This law can be described by only two parameters, e.g., by the values $S_{p}, e_{p}$ at the peak tensile stress, or by $S_{p}$ and the microlevel stiffness $k$ [Fig. 2(a)]. For this law, the energy $\hat{G}_{f}$ needed to break one link, called the microfracture energy and graphically represented as the area under the tensile part of the $S$ $e$ curve, depends on the "microstiffness" $k$ and the "microstrength" $S_{p}$ and is given by $\hat{G}_{f}=S_{p}^{2} / 2 k$. It must be distinguished from the macrofracture energy $G_{f}$ (the usual fracture energy of the material on the macrolevel).

If the stiffness, strength, and toughness of the model are to be controlled independently, a law with three parameters is needed. Its simplest form is a bilinear law with a linear elastic part and a linear softening part [Fig. 2(b)]. If $e_{f}$ denotes the link extension at complete fracture, the microfracture energy can be expressed as $\hat{G}_{f}=S_{p} e_{f} / 2$.

Clearly, if the model is to be isotropic at least in some sense, the properties of the principal links and of the diagonal links must be properly coordinated. Let us denote the parameters of the principal links by a subscript, and those of the diagonals by a subscript ${ }_{2}$. A total of six parameters, three for each group of links, have to be given to fully specify the mechanical properties of the model. The elastic stiffness of the continuum to be modeled, as well as the isotropy requirement, impose certain restrictions on these parameters.

Let us first pay attention to the elastic properties. Consider an isotropic continuum with Young's modulus $E$ and Poisson's ratio $\nu$ in a state of uniform strains $\varepsilon_{x}, \varepsilon_{x}, \gamma_{x y}$ (under plane stress), apply the same strains to the particle model and compare the strain energies. The strain energy of the continuum (accumulated in a square cell with dimensions $L$ by $L$ ) is expressed by

$$
\mathscr{E}_{\mathrm{int}}^{(C)}=\frac{E L^{2}}{2\left(1-\nu^{2}\right)}\left[\varepsilon_{x}^{2}+\varepsilon_{y}^{2}+2 \nu \varepsilon_{x} \varepsilon_{y}+\frac{1-\nu}{2} \gamma_{x y}^{2}\right]
$$

On the other hand, the strain energy of the basic cell in the particle model under the same strain is expressed by

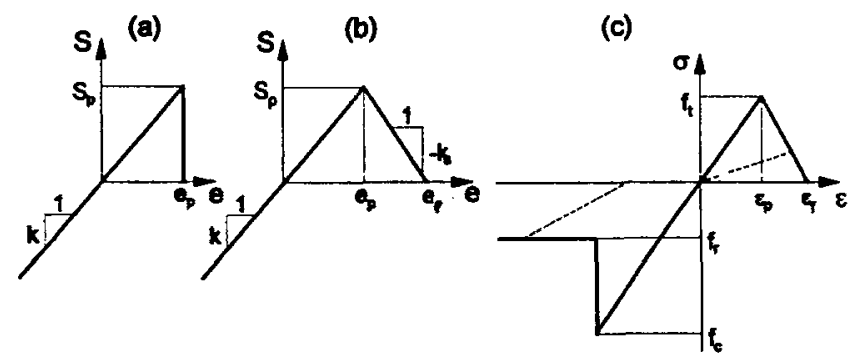

FIG. 2. Simple Microlevel Constitutive Laws: (a) with Sudden Drop; (b) with Linear Softening Part; (c) with Compression Fallure

$$
\begin{aligned}
& \mathscr{E}_{\mathrm{int}}^{(P M)}=\frac{1}{2} \sum_{i=1}^{4} \frac{1}{2} k_{1} e_{i}^{2}+\sum_{i=5}^{6} \frac{1}{2} k_{2} e_{i}^{2} \\
& =\frac{L^{2}}{2}\left[\left(k_{1}+k_{2}\right)\left(\varepsilon_{x}^{2}+\varepsilon_{y}^{2}\right)+2 k_{2} \varepsilon_{x} \varepsilon_{y}+k_{2} \gamma_{x y}^{2}\right]
\end{aligned}
$$

The contribution of the principal links has been divided by 2 because each of them is shared by two neighboring cells. Strain energies $\mathscr{E}_{\text {int }}^{(\mathcal{C})}$ and $\mathscr{E}_{\text {int }}^{(P M)}$ are equal, for any state of strain, if and only if

$$
\frac{E}{2\left(1-v^{2}\right)}=\frac{k_{1}+k_{2}}{2} ; \quad \frac{E v}{\left(1-v^{2}\right)}=k_{2} ; \frac{E}{4(1+v)}=\frac{k_{2}}{2}
$$

Obviously, the last two conditions are in contradiction unless $E v /\left(1-v^{2}\right)=E / 2(1+v)$, which holds only for $v=1 / 3$. If this is the case, $k_{1}$ and $k_{2}$ can be expressed from (5) in terms of Young's modulus $E$ as $k_{1}=3 E / 4$, and $k_{2}=3 E / 8$. To sum up, it has been shown that the square lattice is elastically isotropic if and only if $k_{1}=2 k_{2}$, in which case $v=1 / 3$ and $E=4 k_{1} / 3=8 k_{2} / 3$ always. This agrees with the well-known classical result that Poisson's ratio for random macroscopically isotropic lattices cannot be arbitrarily adjusted and is always equal to $1 / 3$ in two dimensions (and $1 / 4$ in three dimensions), in the limit for sufficiently large systems. Incidentally, $v=0.33$ also happens to be the correct value of Poisson's ratio for sea ice.

The strength and fracture characteristics of the principal and diagonal links should also be somehow related to minimize the directional dependence of the model. The basic requirement is that if the structure fails across a line aligned either with the principal or with the diagonal direction, the maximum force per unit length of the line should be the same in both situations and the same should hold for the energy needed to break the links completely. Of course, these simple conditions do not guarantee the strength or fracture energy to be the same in any direction, but they probably constitute the optimum approximation of isotropy.

Consider a principal failure line $A$ and a diagonal failure line B [Fig. 3(a)]. Line A cuts one principal link and two diagonal links per length $L$ and line $B$ cuts two principal links and two diagonal links per length $\sqrt{2} L$. By comparing the energy needed to completely break the links per unit length of the failure line, we get the condition $\left(\hat{G}_{f 1}+2 \hat{G}_{f 2}\right) / L=$ $\left(2 \hat{G}_{f 1}+2 \hat{G}_{f 2}\right) / \sqrt{2} L$, from which $\hat{G}_{f 2}=\hat{G}_{f 1} \sqrt{2} / 2$.

In a similar way, look at the maximum normal force per unit length that can be transmitted across line $\mathrm{A}$ or $\mathrm{B}$. This is slightly more complicated, because the peak axial force cannot be expected to be reached in all the links cut by the line at the same strain. Consider first the case of uniaxial stress applied in the $x$-direction perpendicular to line $\mathrm{A}$ and denote the principal links in the $x$-direction (loading direction) by 1 and the principal links in the $y$-direction (direction of the failure line) by 3 [Fig. 3(b)]. Equilibrium requires that $\sigma_{x}=\left(S_{1}+\sqrt{2} S_{2}\right) / L, \sigma_{y}=\left(\sqrt{2} S_{2}+S_{3}\right) / L=0$, and com-
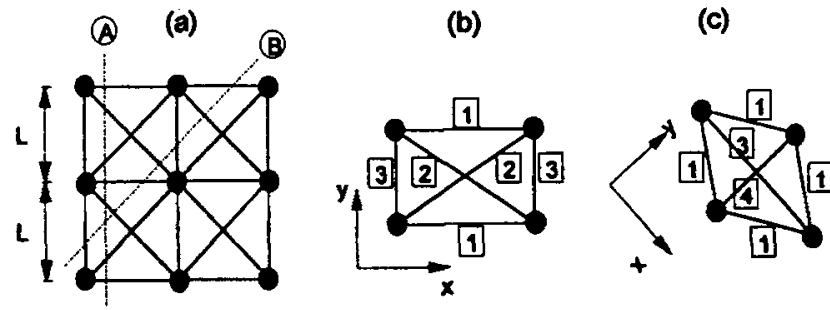

FIG. 3. (a) Principal Failure Line $A$ and Diagonal Failure Line B; (b) Stretching along Principal Direction; (c) Stretching along DIagonal Direction 
patibility requires that $e_{2}=\left(e_{1}+e_{3}\right) / \sqrt{2}$. The links in the $y$-direction are no doubt under compression and, thus, in an elastic state: $S_{3}=k_{1} e_{3}$. Using the last two relations, $S_{3}$ and $e_{3}$ can be eliminated from the foregoing condition of equilibrium in the $y$-direction, which yields $S_{2}=k_{1}\left(e_{1} / \sqrt{2}-e_{2}\right)$. The maximum resistance may be expected to occur when $e_{1}$ $=e_{1 p}, e_{2}<e_{2 p}$, and so $S_{2}=k_{2} e_{2}$. Combining this with the previous expression for $S_{2}$, one gets $S_{2}=S_{1 p} k_{2} \sqrt{2} / 2\left(k_{1}+\right.$ $\left.k_{2}\right)$. Substitution into the expression for $\sigma_{x}$ then yields the final expression

$$
\sigma_{x, \max }=\frac{S_{1 p}}{L} \frac{k_{1}+2 k_{2}}{k_{1}+k_{2}}=\frac{4}{3} \frac{S_{1 p}}{L}
$$

We have used the previously derived relation $k_{1}=2 k_{2}$. The expression derived for the maximum stress transmitted across the line $A$ is valid only under the assumption that after the peak force in link 1 is reached, the stress starts decreasing. This is certainly true if the force $S_{1}$ suddenly drops down but at the same time it is certainly not true if $S_{1}$ softens with a very small slope (the limiting case is ideal plasticity, for which, after sufficient deformation, $S_{1}=S_{1,}$ and $S_{2}=S_{2,}$ simultaneously). It can be shown that the condition for the peak stress to be realized at the peak force in link 1 is $k_{1 s}<-k_{1}$ / 3 , where $k_{1 s}$ is the slope of the softening part of the microlevel constitutive law for principal links.

A similar analysis can be performed for the case of uniaxial stress applied in the direction perpendicular to line B [Fig. $3(\mathrm{c})]$. The result is that

$$
\sigma_{x, \text { max }}=\frac{S_{2 p}}{L} 2 \sqrt{2} \frac{k_{1}+2 k_{2}}{k_{1}+4 k_{2}}=\frac{4 \sqrt{2}}{3} \frac{S_{2 p}}{L}
$$

under the assumption that the peak force is reached in link 2 before it is reached in link 1 , and that the postpeak slope in the microlevel constitutive law for diagonal links $k_{2 s}$ satisfies the condition $k_{2 s}<-k_{1} k_{2} /\left(k_{1}+4 k_{2}\right)=-k_{2} / 3$. The maximum stress levels for failure across line $A$ and across line $\mathrm{B}$ are the same if $4 S_{1, p} / 3 L=4 \sqrt{2} S_{2 p} / 3 L$, which is equivalent to $S_{1 p}=\sqrt{2} S_{2 p}$. Using this information and the relation between the microstiffnesses $k_{1}=2 k_{2}$, the extensions at peak force can be related by $e_{1 p}=e_{2 p} \sqrt{2} / 2$ and it can be verified that the assumed order in which the links attain the peak force was correct.

The conclusion is that if the microlevel constitutive law is not too ductile (in the sense that $k_{i s}<-k_{i} / 3, i=1,2$ ), the peak forces $S_{1 p}, S_{2 p}$ for the principal and diagonal links should be related by $S_{1 p}=\sqrt{2} S_{2 p}$. Along with the relation $\hat{G}_{f 2}=$ $\hat{G}_{f 1} \sqrt{2} / 2$ for the microlevel fracture energy and $k_{1}=2 k_{2}$ for the microstiffness, this provides three conditions restricting the six material parameters of the model.

\section{NUMERICAL STABILITY AND DIRECTIONAL BIAS}

Let us now analyze the numerical stability of the integration method. As already explained, a safe estimate (lower bound) of the critical time step for a linear finite-element model is $2 / \omega_{\max }$, where $\omega_{\max }$ is the largest circular eigenfrequency of the individual elements. The mass matrix of the present model corresponds to the concentrated masses assigned to individual particles. However, the very same matrix could be assembled from fictitious contributions of truss elements with an elemental mass matrix $\mathbf{M}_{e}=m_{e} \mathbf{I}$, where $m_{e}$ represents a properly chosen portion of the particle mass (assigned to the element just for the purpose of the critical time step derivation). The eigenproblem

$$
\begin{aligned}
& \operatorname{det}\left(\mathbf{K}_{e}-\omega^{2} \mathbf{M}_{e}\right)=\operatorname{det}\left|\begin{array}{cc}
k_{e}-\omega^{2} m_{\iota}, & -k_{e} \\
-k_{e}, & k_{e}-\omega^{2} m_{e}
\end{array}\right| \\
& \quad=\left(k_{e}-\omega^{2} m_{e}\right)^{2}-k_{e}^{2}=0
\end{aligned}
$$

yields two circular eigenfrequencies, $\omega_{0}=0$ and $\omega_{c}=$ $\sqrt{2 k_{e} / m_{e}}$. The existence of a zero eigenfrequency is a consequence of the fact that the element is considered to be unsupported. The circular eigenfrequency of interest is $\omega_{e}$. To compute $\omega_{e}$ for the diagonal and principal links, it is necessary to divide the total particle mass $m$ and distribute it among the links connected to it. If the masses assigned to the principal and diagonal links are denoted by $m_{1}, m_{2}$, respectively, then $m=4\left(m_{1}+m_{2}\right)$, as there are four principal and four diagonal links connected to a typical particle. The choice of $m_{1}$ and $m_{2}$ is otherwise arbitrary, but the best lower bound on the critical time step is obtained if the circular eigenfrequencies $\omega_{1}=\sqrt{2 k_{1} / m_{1}}, \omega_{2}=\sqrt{2 k_{2} / m_{2}}$ for the principal and diagonal links are the same. As $k_{1}=2 k_{2}$, the best lower bound is achieved for $m_{1}=2 m_{2}$, which implies that $m_{1}=$ $m / 6, m_{2}=m / 12$. The corresponding estimate of the critical time step is

$$
\Delta t_{c} \geq \Delta t_{c}^{(L)}=\frac{2}{\omega_{1}}=\sqrt{\frac{2 m_{1}}{k_{1}}}=\sqrt{\frac{m}{3 k_{1}}}=\sqrt{\frac{4 \rho L^{2}}{9 g E}}=\frac{2}{3} \frac{\mathrm{L}}{\mathrm{c}}
$$

where $c=\sqrt{E / \rho}=$ longitudinal wave velocity (speed of sound) in ice. Eq. (9) is a safe lower bound even though, at the boundary of the particle system, the particle mass must be distributed among fewer than eight links. However, a larger element mass matrix results in a lower eigenfrequency and, therefore, $\omega_{1}, \omega_{2}$ as the circular eigenfrequencies of the typical links in the interior of the system are maximum among all the links.

The usual consequence of a numerical instability is a fast exponential growth of displacements, quickly resulting in a numerical overflow. But this is the case only for linear systems and for those nonlinear systems that can carry stresses even after very large deformations, e.g., for geometrically nonlinear elastic models. However, a fracturing material model totally breaks apart during the initial stage of a stability loss and, after the failure of all links, no internal forces can be produced any more and the individual broken pieces travel at a constant velocity (unless the external forces accelerate them). It should be emphasized that this type of instability manifestation can be difficult to recognize in situations where some kind of failure is expected. Especially if the time step is only slightly above the critical one and the integration is performed only over a relatively short time interval, the instability might not completely destroy the system and the result could be erroneously attributed to real physical failure mechanisms. In conclusion, special attention must be paid to the correct assessment of the critical time step when dealing with mechanical systems that do not carry any stresses if the strains are sufficiently large.

The next problem to examine is that of mesh-induced directional bias, which has usually been ignored in the literature and, as we will see, is inevitable and severe. To minimize the influence of the floe shape, we will consider a circular floe instead of a rectangular floe and study a central impact of the floe on a cylindrical obstacle. The floe is divided into particles arranged in a regular square lattice. The attack angle $\alpha$, defined as the angle between the principal lattice direction and the direction of impact, was varied between $0^{\circ}$ and $45^{\circ}$. All the other parameters were the same for all simulations: floe radius $r_{f}=100 \mathrm{~m}$, obstacle radius $r_{0}=100 \mathrm{~m}$, particle spacing $L=5 \mathrm{~m}$, impact velocity $v=1 \mathrm{~m} / \mathrm{s}$ and integration step $\Delta t=10^{-3} \mathrm{~s}$ (safely below the stability limit). The details of the modified microlevel constitutive law will be explained in the following section along with the reasons for the changes as compared to the previously considered simple bilinear law, but the law and the material parameters were essentially the same as those in the final simulations with a random geometry, which led to satisfactory results. The only difference was 


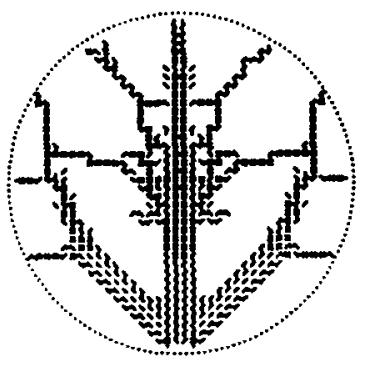

(c)

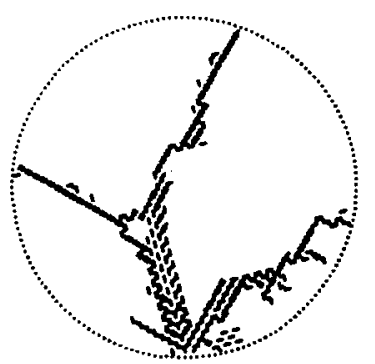

FIG. 4. Failure Patterns for Various Values of $\alpha$ : (a) $0^{\circ}$; (b) $15^{\circ}$; (c) $30^{\circ}$; (d) $45^{\circ}$ (Model with Deterministic Strength)

that the microfracture energy was slightly larger in the bias study to emphasize the fracture process zones.

Fig. 4 shows the failure patterns $1 \mathrm{~s}$ after the impact for four different values of the attack angle. It is clear that the orientation of the principal direction, which is a subjective matter of choice, has an enormous effect on both the direction and the width of the zones of intense damage. The short lines plotted in the figures are perpendicular to the directions of broken links and can be thought of as cracks. Clearly, the cracks prefer to propagate along the directions of either the principal links or the diagonal links. Directions inclined with respect to the preferred ones are rarely followed by the cracks. This results in strongly asymmetric failure patterns when the impact direction is not aligned with any of the preferred directions (e.g., if $\alpha=15^{\circ}$ or $\alpha=30^{\circ}$ ). On the other hand, for $\alpha=0^{\circ}$ and $\alpha=45^{\circ}$, the failure patterns are almost perfectly symmetric but widely different from each other. In addition, the fracture process zones are much wider when the directions are aligned than when they are not aligned.

The problem with directional bias persists even when the strength of the links is randomized. This is shown by another set of numerical simulations with random strength. The strength had a log-normal distribution with the coefficient of variation being $30 \%$. The floe was larger than in the previous example $\left(r_{f}=250 \mathrm{~m}\right)$ and only three angles of attack were used. In spite of the substantial randomness, the failure patterns in Fig. 5 indicate that the cracks again tend to propagate mainly along the principal directions.

It must be concluded that the regular lattices lead to biased results and, therefore, should not be used to simulate isotropic solids. Although we studied only the square lattice, it can be expected that the triangular lattice, which has only three basic directions as compared with four for the square pattern, would exhibit an even stronger directional bias. A hexagonal lattice is more or less a variant of the triangular lattice.

\section{PARTICLE MODEL WITH RANDOM GEOMETRY}

To obtain a directionally unbiased model, it is proposed to generate the coordinates of particle centers in a random way. (a)

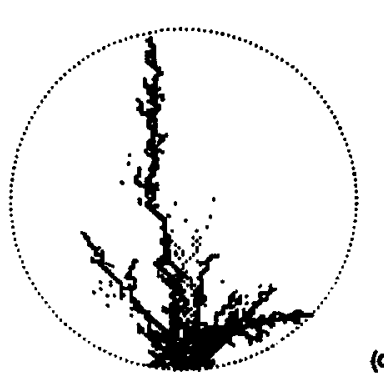

(c)
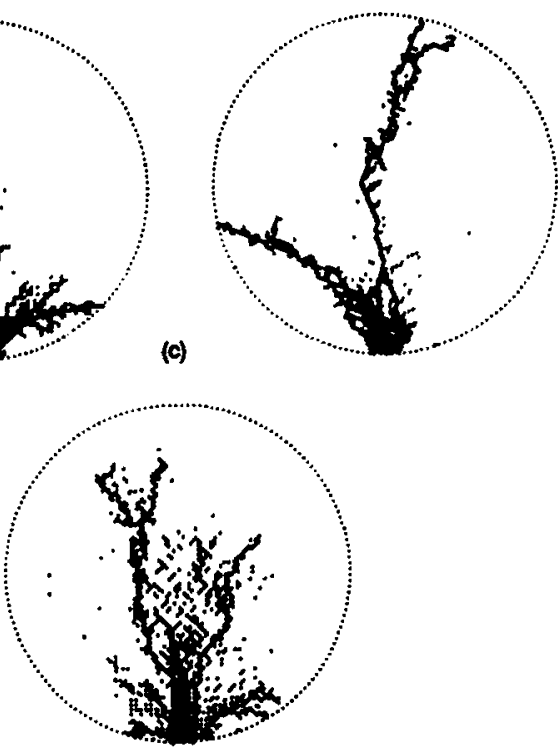

FIG. 5. Failure Patterns for Various Values of $\alpha$ : (a) $0^{\circ}$; (b) $22.5^{\circ}$; (c) $45^{\circ}$ (Model with Random Strength)

A uniform probability density over the floe area is used, with a restriction of a certain minimum distance $L_{\min }$ between any two neighboring particle centers. $L_{\min }$ should be chosen such that an excessive local concentration of particles be suppressed. Denoting the floe area by $A_{f}$, one can define a nondimensional parameter $\lambda_{1}=L_{\min } / \sqrt{A_{f} / n}$ as a measure of relative particle density. The value of $\lambda_{1}=1$ corresponds to a regular square lattice. It has been found that $\lambda_{1}=0.8$ still leads to an acceptable macroscopic uniformity of the particle arrangement and this value is used for the present study.

Interaction between two particles is assumed to take place if their centers are closer than a certain interaction distance, $L_{\max }$. This form of the interaction rule is easy to implement and it generates reasonable link structures when a proper value of the ratio $\lambda_{2}=L_{\text {max }} / L_{\text {min }}$ is chosen. A good choice seems to be, e.g., $\lambda_{2}=2$.

The present approach to generating a random lattice differs from the one developed by Moukarzel and Herrmann (1992) and applied by Schlangen (1993), in which a regular square array of nodes is perturbed and subsequently connected by links using the Voronoi construction. The resulting lattice is irregular but some directional preferences still persist. Therefore, the approach suggested here seems to be safer.

The macroscopic Young's modulus of the lattice can be adjusted by a proper choice of the microstiffnesses. In the present model, all the links are assumed to have the same cross-sectional stiffness $E A$, where $E$ is Young's modulus of the macroscopic continuum and $A$ is the effective area of the link to be determined from the condition that the macroscopic Young's modulus of the lattice also be $E$.

The easiest way to determine the macroscopic and microscopic stiffness of the model is to consider a state of equibiaxial plane stress $\left(\sigma_{x}=\sigma_{y}=\sigma\right.$, with $\left.\varepsilon_{x}=\varepsilon_{y}=\varepsilon\right)$, and compare the strain energies of the particle model and of an equivalent continuum, with Young's modulus $E$ and Poisson's ratio $v=1 / 3$. Equating the strain energies of the floe and the particle model, we have $E A_{f} \varepsilon^{2} /(1-v)=\sum_{i=1}^{n} E A L_{i} \varepsilon^{2}$ 2. Therefore, $A=3 A_{f} / \Sigma_{i=1}^{n} L_{i}$, where $L_{i}, i=1,2, \ldots n$, are the lengths of the links.

The microlevel constitutive equation (force-displacement law) for an individual link must be as simple as possible to ensure numerical efficiency. Simultaneously, it must reflect the essential mechanical properties of the material. Instead 

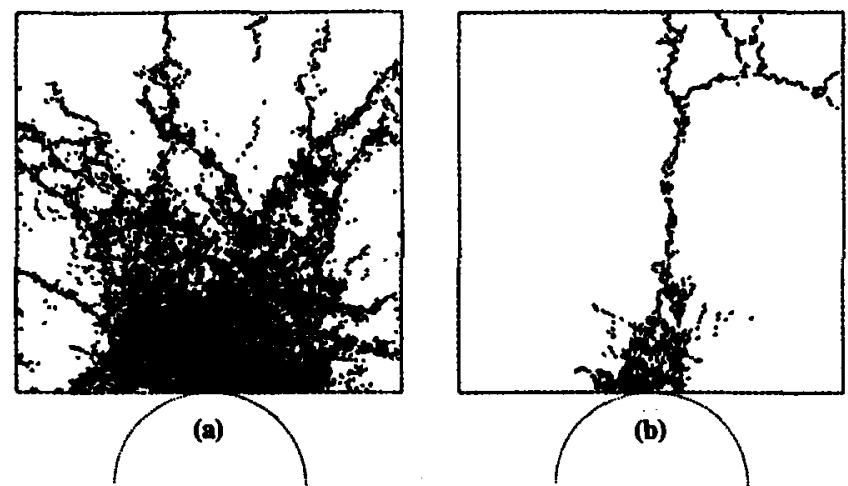

FIG. 6. Crack Pattern: (a) without Compression Fallure; (b) with Compression Fallure

of the bilinear force-displacement law we used before, for the model with a random geometry it is more convenient to describe the constitutive relations in terms of stress and strain in the link. This is possible using the effective cross-sectional area $A=3 A_{f} / \Sigma_{i=1}^{\prime \prime} L_{i}$. The axial force $S_{i}$ in link number $i$ can be replaced by a microstress $\sigma_{i}=S_{i} / A$ and the extension of the link $e_{i}$ by a microstrain $\varepsilon_{i}=e_{i} / L_{i}$. The bilinear microscopic stress-strain law is then described by three parameters: Young's modulus $E$, microstrength $f_{n}$, and microstrain at complete failure $\varepsilon_{f}$. The microscopic fracture energy of the $i$ th link $\hat{G}_{f i}$ is now given by

$$
\hat{G}_{f i}=\frac{1}{2} A L_{i} f_{i} \varepsilon_{f}
$$

A more reasonable possibility, used before by Bažant et al. (1990), would be to assume that $\hat{G}_{f}$ for each link is a basic microlevel parameter, in which case $\varepsilon_{f}$ is variable and $\varepsilon_{f}=$ $2 \hat{G}_{f} /\left(A L_{i} f_{i}\right)$. This corresponds to the assumption that the link fails by a single crack, with the same fracture energy. However, this description is numerically inconvenient because, for a long $L_{i}$ and not too large $\hat{G}_{f}$, it can yield $\varepsilon_{f}$ smaller than the strain at peak stress, which causes snapback instabilities of the links and complicates numerical solution. For this reason, (10) is preferred here. The difference is probably unimportant for the macrobehavior.

The expression "microscopic" refers, in the present context, to the scale of one link as compared with the entire model, even though in the ice floe each particle will represent an area of several square meters, which is large compared with the laboratory scale. It is thus necessary to adjust the values of material parameters determined on laboratory specimens; the strength should be decreased and the fracture energy should be increased, because large inhomogeneities cause the fracture process zone to be much larger than in the laboratory. In this study, the parameters have been set to $E=$ $5 \mathrm{GPa}, f_{t}=0.4 \mathrm{MPa}$, and $\varepsilon_{f}=80 \times 10^{-6}$, which corresponds

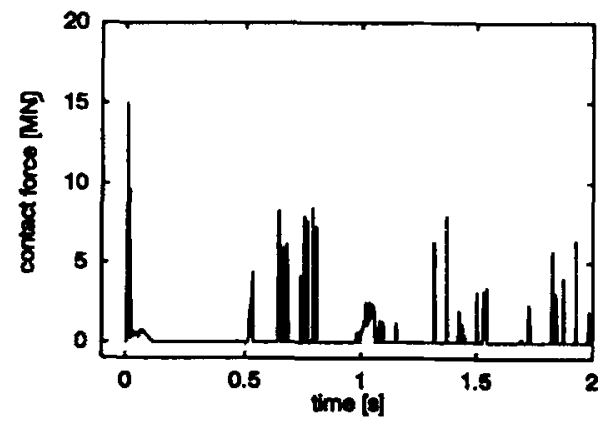

(a) to a vertical drop in the microlevel stress-strain diagram $\left(\varepsilon_{f}\right.$ $=\varepsilon_{t}$ ). The fracture energy per unit area of a broken link $i$ is equal to $\hat{G}_{f i}$ divided by the effective area $A$. This yields for an average length $L_{i}=6 \mathrm{~m}$ the value $96 \mathrm{~J} / \mathrm{m}^{2}$, which is much larger than the laboratory value of $2 \mathrm{~J} / \mathrm{m}^{2}$.

The initial simulations of the central impact of a square ice floe on a rigid cylindrical obstacle were run with the bilinear law for tension and with a linear elastic law in compression. It turned out, however, that this type of model leads to an almost complete destruction of the ice plate even for moderate initial velocities [Fig. 6(a)]. The reason is that if the only mechanism dissipating energy is the tensile fracture of links, a large amount of potential energy is stored during the impact in compressed links in the impact region and when subsequently released, it is transmitted by waves across the floe, which results in tensile fracture almost everywhere. A simple comparison of the total kinetic energy $\mathscr{C}_{k}=A_{f} \rho v^{2 / 2}$ and the energy needed to break all links, $\mathscr{E}_{f}=\sum_{i=1}^{n} \hat{G}_{f i}=$ $0.5 A f_{t} \varepsilon_{f} \sum_{i=1}^{n} L_{i}=1.5 A_{f} f_{i} \varepsilon_{f}$, shows that, for density $\rho=$ $910 \mathrm{~kg} / \mathrm{m}^{3}$ and initial velocity $v=1 \mathrm{~m} / \mathrm{s}, \mathscr{E}_{k}$ is ten times larger than $\mathscr{E}_{f}$. In other words, as little as $10 \%$ of the kinetic energy is sufficient to destroy all links. Although the floe does not have to lose all its kinetic energy during the impact event, energy dissipation mechanisms other than the tensile fracture must necessarily be taken into account.

Observations show that an intensive crushing usually takes place in the contact region between the floe and the obstacle. This type of failure can be modeled by modifying the stressstrain law for compression. Linear softening similar to the law introduced for tension would be unrealistic. Whenever a particle would hit the obstacle, the contact force would blow up and subsequently abruptly drop down to zero when the compressed links connecting this particle fail [Fig. 7(a)]. It is clear that even after a part of the floe has crushed, the ice debris can still resist some force while it is pushed up or down. It is proposed to model the corresponding forces by a horizontal plateau in the compressive part of the stress-strain diagram. The complete stress-strain diagram used in this study is plotted in Fig. 2(c). In addition to the parameters already introduced for tension, two stresses $f_{c}, f_{r}$ are necessary to define the compressive behavior. Fig. 2(c) also specifies the unloading behavior both for tension and for compression. Hysteresis at unloading-reloading cycles is replaced by a linear viscous damping element coupled in parallel with the fracturing element. This means that the total stress is a sum of a strain-dependent term $\sigma_{f}$ according to Fig. 2(c) and a viscous term $\sigma_{v^{\prime}}=\eta \dot{\varepsilon}$ proportional to the strain rate. Further, the viscous term also takes, approximately, into account the creep of ice. The combination of compression yield and viscous damping gives realistic results in terms of both the crack patterns [Fig. 6(b)] and the contact force history [Fig. 7(b)].

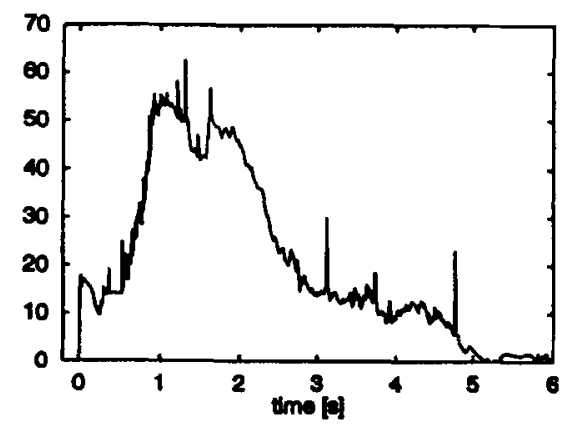

(b)

FIG. 7. Contact Force: (a) without Residual Stress in Compression; (b) with Residual Stress in Compression 

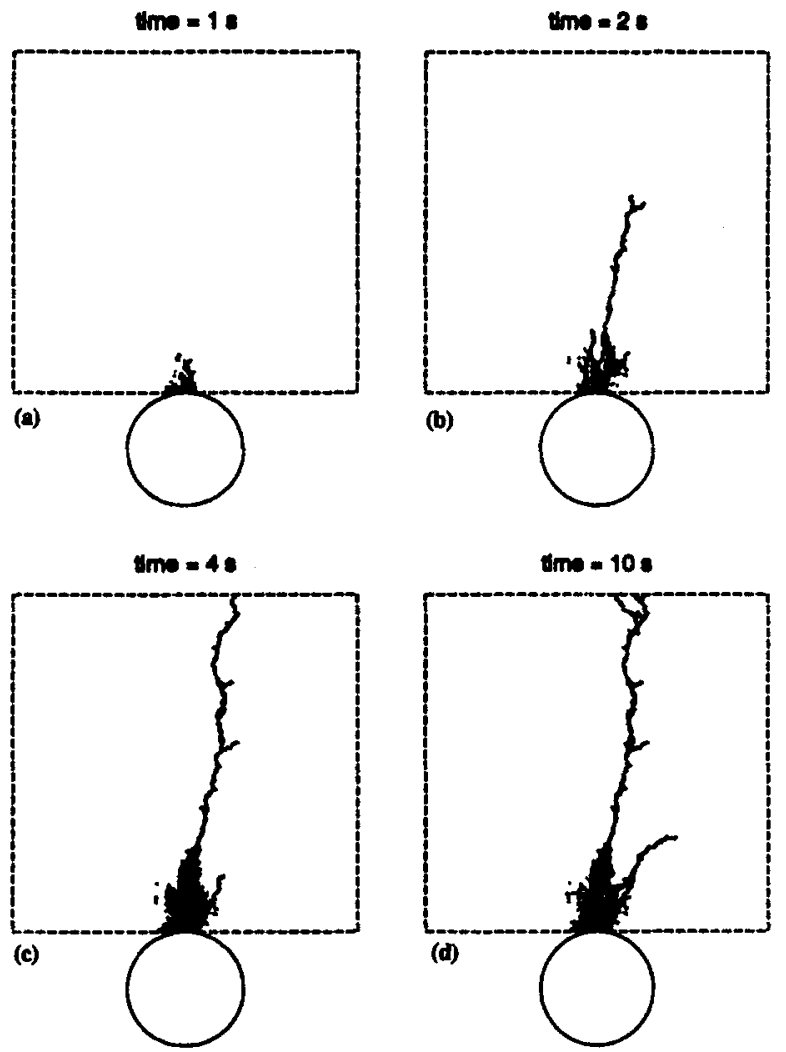

FIG. 8. Evolution of Tenslle Fracture

\section{NUMERICAL RESULTS}

The model was implemented in an explicit dynamic code and was used to simulate the impact of a square ice floe on a rigid cylindrical obstacle. The floe size varied between 100 $\times 100 \mathrm{~m}$ and $1,200 \times 1,200 \mathrm{~m}$. All the thickness-dependent quantities such as the mass of a particle or the contact force were related to $1 \mathrm{~m}$ of thickness. One particle always represented $25 \mathrm{~m}^{2}$ of the floe, and so the total number of particles varied between 400 and 57,600 . The integration of 15,000 time steps for the largest model with 115,200 degrees of freedom took about two days on model 710 of the Hewlett-Packard Apollo 9000 workstation.

The particles were randomly generated using $L_{\min }=4 \mathrm{~m}$, $L_{\max }=8 \mathrm{~m}$, and $L=\sqrt{A_{f} / n}=5 \mathrm{~m}$. The material parameters used in most of the simulations were $E=5 \mathrm{GPa}, f_{t}=0.4$ $\mathrm{MPa}, \varepsilon_{f}=80 \times 10^{-6}, f_{c}=-2.4 \mathrm{MPa}, f_{r}=-1.2 \mathrm{MPa}, \eta$ $=5 \mathrm{MPa} \mathrm{s}$, and $\rho=1,000 \mathrm{~kg} / \mathrm{m}^{3}$. The lower bound on the critical time step based on an analogy with finite elements is now slightly more difficult to construct; because the geometrical arrangement is not regular, every link has a different stiffness and the number of links connected to a particle varies. For a safe estimate, one can consider the worst case of maximum possible stiffness and minimum possible mass combined in one link. The maximum stiffness is given by $k_{\max }=$ $E A / L_{\min }$ and the minimum mass is given by $m_{\min }=m / n_{\max }$, where $n_{\text {max }}$ is the maximum number of links connected to one particle. The effective area $A$ varies slightly depending on the floe size and the particular random realization but it never exceeds $A_{\max }=8 \mathrm{~m}$ in the simulations discussed here. The maximum number of links connected to one particle was found to be $n_{\max }=8$. Substituting these values into $\Delta t_{c}^{(L)}=2 / \omega_{\max }$, the lower bound on the critical time step is constructed

$$
\begin{aligned}
& \Delta t_{c}^{(\ell)}=\sqrt{\frac{2 m_{\min }}{k_{\max }}}=\sqrt{\frac{2 p L^{2} L_{\min }}{n_{\max } E A_{\max }}}=\frac{L}{c} \sqrt{\frac{2 L_{\min }}{A_{\max } n_{\max }}} \\
& \quad=1.118 \times 10^{-3} \mathrm{~s}
\end{aligned}
$$

Numerical tests confirmed that $\Delta t=10^{-3} \mathrm{~s}$ was a stable time step and this value was used in all the computations.

Various stages of a typical impact event are documented
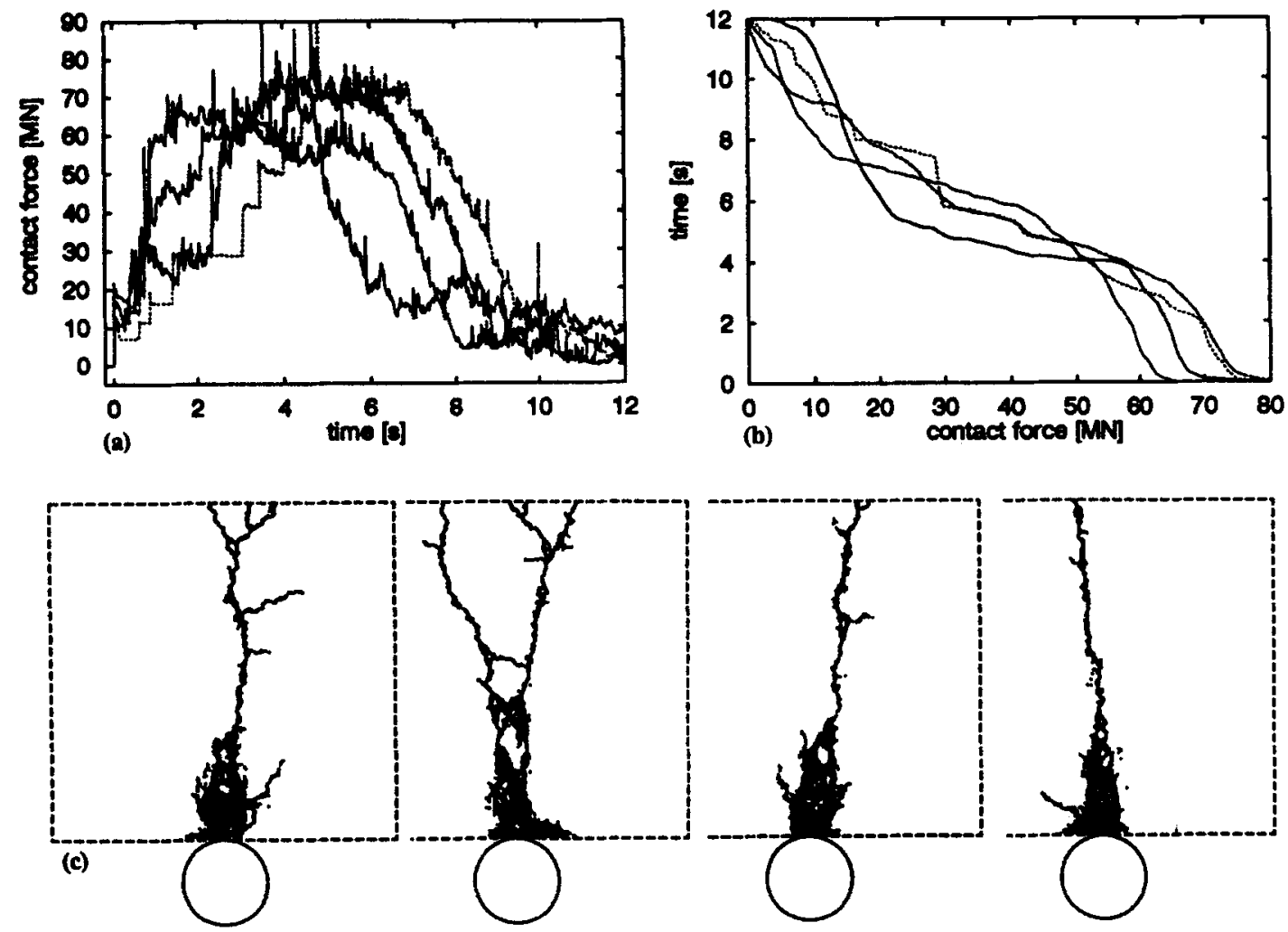

FIG. 9. (a) Contact Force History for Different Realizations of Same Event; (b) Time Spent above Given Contact Force Level for Different Realizations of Same Event; (c) Cracking Patterns for Different Realizations of Same impact 

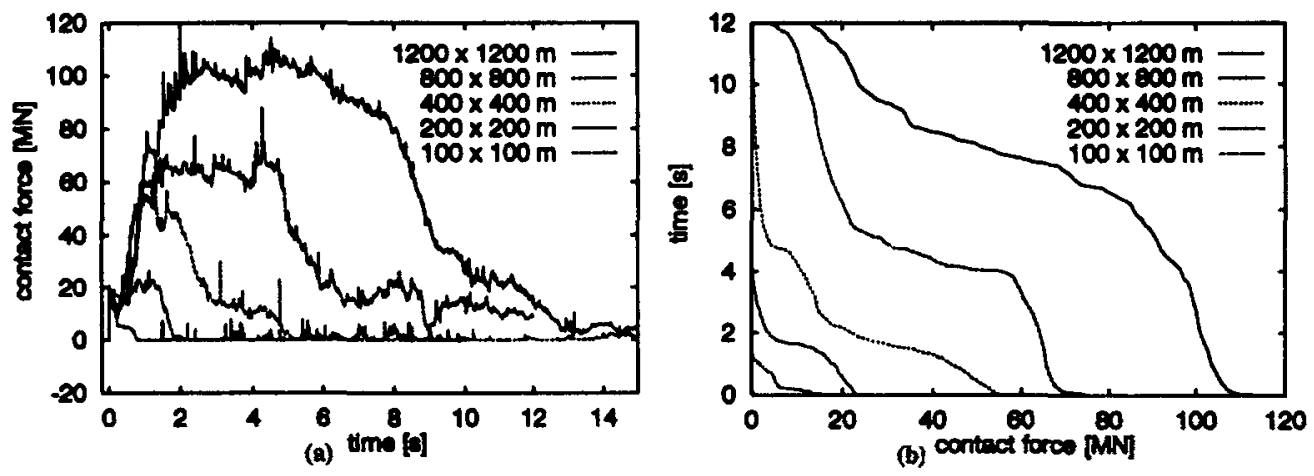

FIG. 10. (a) Contact Force History for Floes of Different Slzes; (b) Time Spent above Glven Contact Force Level for Floes of Different Sizes
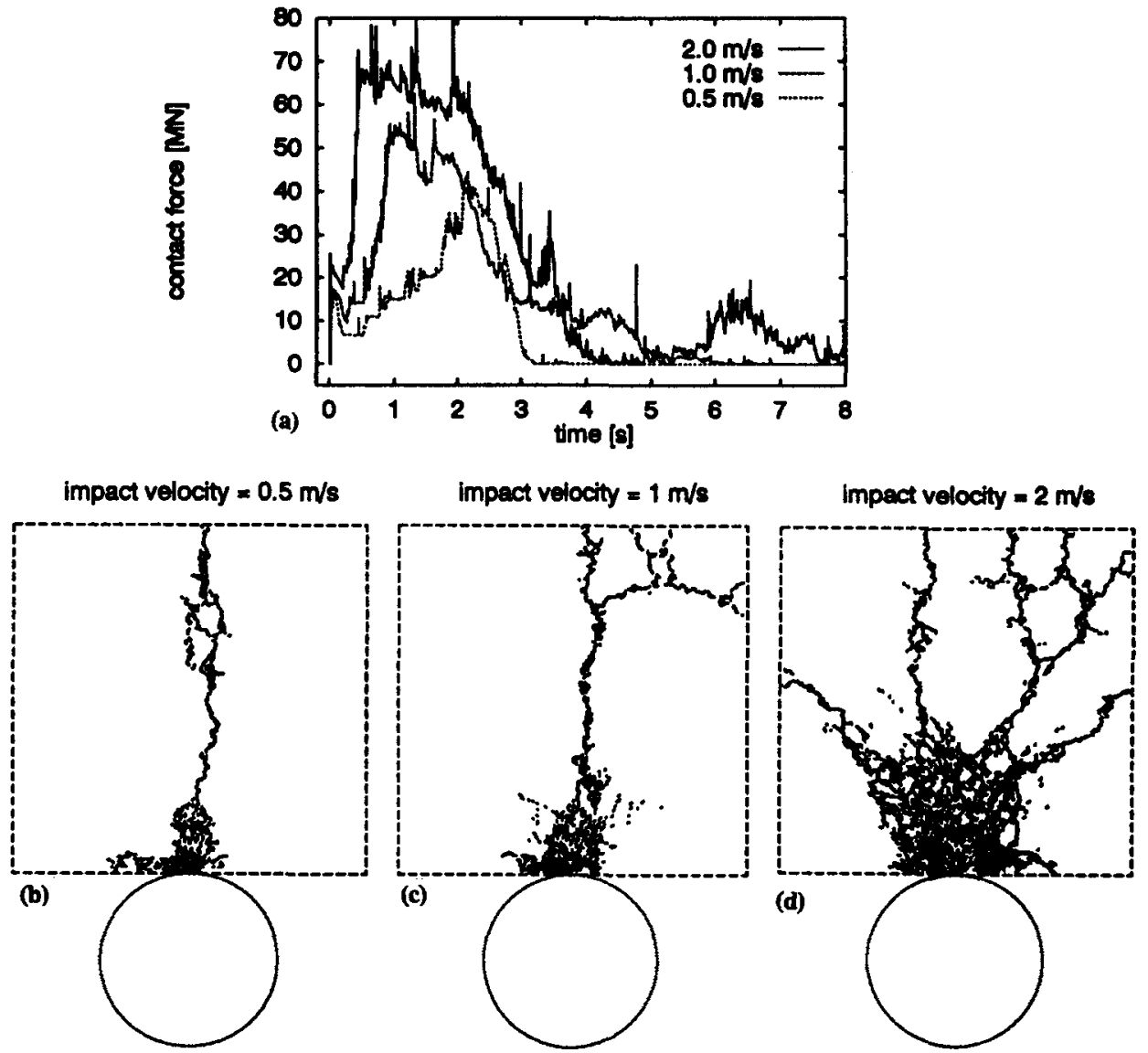

FIG. 11. Contact Force History and Cracking Patterns for Different Impact Velocities

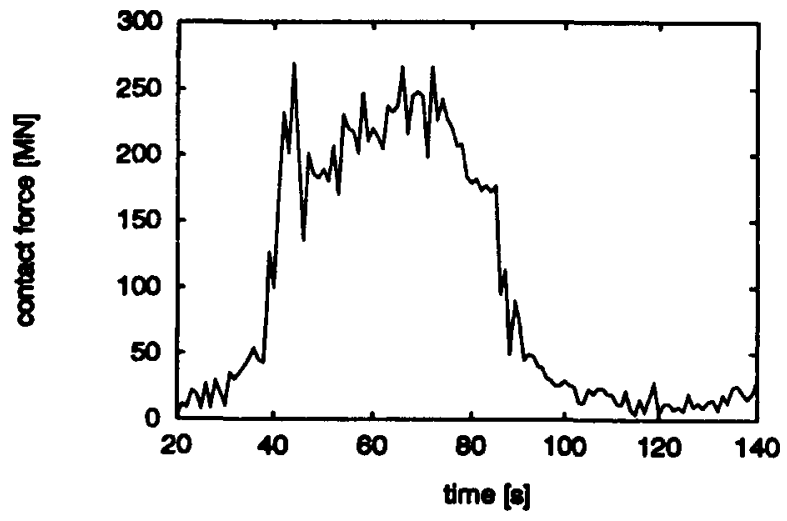

FIG. 12. Contact Force History for Event 2 at Hans Island, 4 August 1981 in Fig. 8. This example refers to the largest floe of $1,200 \times$ $1,200 \mathrm{~m}$ in size, hitting an obstacle with the radius $r_{0}=200$ $\mathrm{m}$ at the initial velocity $v=1 \mathrm{~m} / \mathrm{s}$. In spite of the strong influence of the compressive part of the stress-strain law on the overall performance of the model, the number of links damaged by crushing is very small and the damaged links are concentrated in the contact area.

To get an idea about the scatter of the numerical results due to the randomness of the geometrical arrangement, the impact of the floe $800 \times 800 \mathrm{~m}$ at the initial velocity $v=1$ $\mathrm{m} / \mathrm{s}$ was simulated for four different random realizations. The contact force histories are plotted in Fig. 9(a). As seen, the curves differ locally but their most important overall characteristics remain almost the same. This is true not only for the maximum force but also for the total time during which the contact force exceeds a chosen value. This result appears to be satisfactory because the specifics of the contact force 
history on real floes no doubt depend on random factors; e.g., the initial stage is strongly affected by the condition of the ice in a small region close to the first contact point rather than by the global characteristics of the floe. These are reflected only in the global behavior of the contact force, which is captured by the model in a reliable way.

Instead of plotting the contact force history, the total time during which a given level of the contact force was exceeded can be plotted as a function of the contact force level [Fig. 9(b)]. The curves corresponding to the different random realizations are much closer in this plot than in Fig. 9(a), which supports the statement that the global characteristics of the behavior are captured better than the local specifics of the contact force history. This is further evidenced by Fig. 9(c), which shows various cracking patterns calculated for different realizations of the same impact.

Fig. 10(a) presents a comparison of the contact force histories for five different floe sizes, with $r_{o}=100 \mathrm{~m}$ and $v=$ $1 \mathrm{~m} / \mathrm{s}$. Not only the peak force but also the total duration of the contact increases with the size of the floe. This trend is especially important with regard to model verification, because the observed impact events for large floes (many square kilometers in size) often extend over a period of several minutes. The alternative plot of the total time above a given contact force level in Fig. 10(b) emphasizes the trend toward longer impact times for larger floes.

Fig. 11 shows the effect of the initial velocity for the floe dimensions $400 \times 400 \mathrm{~m}$ and the obstacle radius $r_{a}=100$ $\mathrm{m}$. As expected, slower impact velocities result in a more gradual buildup of the contact force. The corresponding cracking patterns are also shown in Fig. 11.

The reported numerical results should, of course, be compared with experimental observations and measurements. Unfortunately, the experimental data available in the public domain are rather scarce, including mainly those observed at Hans Island in 1981. The contact force recorded on August 4,1981 , during the impact of a single floe of area $5.7 \mathrm{~km}^{2}$ and thickness $8 \mathrm{~m}$ at an initial velocity of $0.33 \mathrm{~m} / \mathrm{s}$ is reproduced in Fig. 12 [after Danielewicz and Metge (1981); also see Sanderson (1988)]. A qualitative agreement of the overall shape and character of the measured force history with the preliminary numerical results can be observed. However, as the obstacle was not a cylinder, other failure mechanisms such as flexural failure were no doubt also involved. Moreover, no pictures of the cracking patterns for this or similar events seem to be available in the public domain. No effort has therefore been made to adjust the model parameters so as to reproduce the recorded data quantitatively. The aim of this study is to present a general methodology that can be refined in the future, after more detailed experimental evidence has been made available.

\section{CONCLUSIONS}

1. The particle model (discrete element method) can effectively simulate the fracture of quasibrittle materials with large zones of distributed cracking. Even if the material does not have a particulate structure, the model is a convenient device to endow a strain-softening material with a characteristic length that serves as a localization limiter, similar to nonlocal continuum models. The particle size reflects the maximum size of inhomogeneities in the material.

2. The fracture simulation of a circular specimen is a good test of isotropy.

3. Although a regular lattice of particles can closely approximate isotropic elastic and strength properties, it cannot closely approximate the fracture behavior of iso- tropic material. Even if the microstrength values are randomized, there is a strong directional bias favoring propagation along the lines of the regular lattice. A randomly generated particle system, on the other hand, approximates isotropic fracture properties well.

4. Simple estimates of the critical time step at the limit of numerical stability of an explicit algorithm are derived, and the deceptive way in which fracturing can obscure numerical instability is pointed out.

5. The random particle model gives realistic results in simulating the fracture of a large moving sea ice floe hitting an obstacle.

\section{ACKNOWLEDGMENT}

Partial financial support from Office of Naval Research under Grant N00014-91-2-1109 to Northwestern University is gratefully acknowledged.

\section{APPENDIX. REFERENCES}

Bathe, K. J. (1982). Finite element procedures in engineering analysis. Prentice-Hall, Inc., Engelwood Cliffs, N.J.

Bažant, Z. P., Tabbara, M. R., Kazemi, M. T., and Pijaudier-Cabot, G. (1990). "Random particle model for fracture of aggregate or fiber composites." J. Engrg. Mech., ASCE, 116(8), 1686-1705.

Belytschko, T. B., Phesha, M., and Dowding, C. H. (1984). "A computer method for stability analysis of caverns in jointed rock." Int. J. for Numerical and Analytical Methods in Geomech., Vol. 8, 473-492.

Cundall, P. A. (1971). "A computer model for simulating progressive large scale movements in blocky rock systems." Proc., Int. Symp. on Rock Fracture, ISRM, Nancy, France

Cundall, P. A., and Strack, O. D. L. (1979). “A discrete numerical model for granular assemblies." Geotechnique, London, England, Vol. $29,47-65$.

Danielewicz, B. W., and Metge, M. (1981). "Ice forces on Hans Island." APOA Proj. No. 181, British Petroleum Co., London, England.

Disorder and fracture. (1990). J. C. Charmet, S. Roux, and E. Guyon, eds., Plenum Publishing Corp., New York, N.Y.

Dowding, C. H., Zubelewicz, A., O'Connor, K. M., and Belytschko, T. B. (1991). "Explicit modeling of dilation, asperity degradation, and cyclic seating of rock joints." Comp. and Geotechnics, Vol. 11, 209227

Herrmann, H. J. (1991). "Patterns and scaling in fracture." Fracture processes in concrete, rock and ceramics, Chapmann \& Hall Publishers, New York, N.Y.

Jirásek, M., and Bažant, Z. P. (1995). "Macroscopic fracture characteristics of random particle systems." Int. J. Fracture, Vol. 69, 201228

Kawai, T. (1980). "Some considerations on the finite element method." Int. J. for Numerical Methods in Engrg., Vol, 16, 81-120.

Moukarzel, C., and Herrmann, H. J. (1992). "A vectorizable random lattice." Preprint HLRZ 1/92, HLRZ KFA, Jülich, Germany.

Plesha, M. E., and Aifantis, E. C. (1983). "On the modeling of rocks with microstructure." Proc., 24th U.S. Symp. Rock Mech., Texas A\&M Univ. College Station, Tex.

Plesha, M. E. Hutson, R. W., and Dowding, C. H. (1991). "Determination of asperity damage parameters for constitutive models of rock discontinuities." Int. J. for Numerical and Analytical Methods in Geomech Vol 15, 289-294.

Proceedings of the 11th IAHR international symposium on ice. (1992) T. M. Hrudey et al., eds., Univ. of Alberta, Edmonton, Canada.

Sanderson, T. J. O. (1988). Ice mechanics risks to offshore structures. Graham and Trotman, Boston, Mass.

Schlangen, E. (1993). "Experimental and numerical analysis of fracture processes in concrete," PhD thesis, Tech. Univ. of Delft, The Netherlands

Schlangen, E., and van Mier, J. G. M. (1992). "Experimental and numerical analysis of micromechanisms of fracture of cement-based composites." Cement and Concrete Composites, Vol. 14, 105-118.

Schlangen, E., and van Mier, J. G. M. (1993). "Fracture modeling of granular materials." Proc., Computational Methods in Mat. Sci., Mat. Res. Soc., Pittsburgh, Pa, 153-158.

Serrano, A. A., and Rodriguez-Ortiz, J. M. (1973). "A contribution to the mechanics of heterogeneous granular media." Proc., Symp. Plas. ticity and Soil Mech., Cambridge, England.

Statistical models for the fracture of disordered media. (1990). H. J. Herr- 
mann and S. Roux, eds., North-Holland Publishing Co., New York, N.Y.

Strikwerda, J. C. (1988). Finite difference schemes and partial differential equations. Wadsworth and Brooks, Pacific Grove, Calif.

Thornton, C., Kafui, D.-K., and Yin, K.-K. (1993). "Applications of DEM to impact fracture/fragmentation." Proc., 2nd Int. Conf. on Discrete Element Methods, J. R. Williams and G. G. W. Mustoe, eds., IESL Publications, Cambridge, Mass., 265-273.

van Mier, J. G. M., and Schlangen, E. (1993). "An experimental and numerical study of mode I (tensile) and mode II (shear) fracture in concrete." $J$. of the Mechanical Behavior of Materials, Vol. 4, 179-190.
Zubelewicz, A. (1980). "Contact element method," PhD thesis, Tech. Univ. of Warsaw, Warsaw, Poland (in Polish).

Zubelewicz, A. (1983). "Proposal of a new structural model for concrete." Archiwum Inzynierii Ladowej, Warsaw, Poland, Vol. 29, 417429 (in Polish).

Zubelewicz, A., and Mróz, Z. (1983). "Numerical simulation of rockburst processes treated as problems of dynamic instability." Rock Mech and Engrg., Vol. 16, 253-274.

Zubelewicz, A., and Bažant, Z. P. (1987), "Interface element modeling of fracture in aggregate composites." J. Engrg. Mech., ASCE, Vol. $113,1619-1630$ 